\title{
Esophageal Candidiasis as the Initial Manifestation of Acute Myeloid Leukemia
}

\author{
Yukiko Komeno ${ }^{1}$, Hideki Uryu ${ }^{1}$, Yuko Iwata ${ }^{1}$, Yasumasa Hatada ${ }^{2}$, Jumpei Sakamoto ${ }^{3}$, \\ Kuniko Iihara ${ }^{4}$ and Tomiko Ryu ${ }^{1}$
}

\begin{abstract}
A 47-year-old woman presented with persistent dysphagia. A gastroendoscopy revealed massive esophageal candidiasis, and oral miconazole was prescribed. Three weeks later, she returned to our hospital without symptomatic improvement. She was febrile, and blood tests showed leukocytosis $(137,150 / \mu \mathrm{L}$, blast $85 \%)$, anemia and thrombocytopenia. She was diagnosed with acute myeloid leukemia (AML). She received chemotherapy and antimicrobial agents. During the recovery from the nadir, bilateral ocular candidiasis was detected, suggesting the presence of preceding candidemia. Thus, esophageal candidiasis can be an initial manifestation of AML. Thorough examination to detect systemic candidiasis is strongly recommended when neutropenic patients exhibit local candidiasis prior to chemotherapy.
\end{abstract}

Key words: Candida, esophageal candidiasis, leukemia, neutropenia, gastroendoscopy

(Intern Med 54: 3087-3092, 2015)

(DOI: 10.2169/internalmedicine.54.4613)

\section{Introduction}

Candida species are a component of the endogenous microbiome. Once the cutaneous or mucosal barrier is disrupted and the immune system is impaired, they can become opportunistic pathogens causing local or systemic invasive infections. The cutaneous or mucosal barrier may be breached by mucositis or the use of central venous catheters. The immune system is impaired in patients undergoing chemotherapy, stem cell/organ transplantation, and immunosuppressive therapy. Acquired immune deficiency syndrome (AIDS) causes a decrease in the number of CD4+ helper T cells (1-3). Patients in intensive care units are also at high risk due to poor general conditions with surgical wounds and multiple intravenous catheters $(1,3)$.

A patient's innate immunity is the first line of defense against Candida infections (4). Neutrophils and monocytes/ macrophages are the major cell types which perform phagocytosis (4). Acquired immune system (T cells) and humoral immunity (serum gammaglobulin and mucosal $\operatorname{IgA}$ secretion by $\mathrm{B}$ cells) also play roles in controlling Candida infections $(2,5)$.

Candida infections are classified into 3 categories depending on their localization: cutaneous (the skin and its appendages), mucosal (oropharyngeal, esophageal, and valvovaginal) and systemic (bloodstream infections, including candidemia and other forms of invasive candidiasis) (6). Esophageal candidiasis is an AIDS-defining condition, usually occurring in patients with low CD4+ T cells $(<200$ cells $/ \mu \mathrm{L}$ ) (2). Multiple cultures (blood, urine, cerebrospinal fluid, and valvovaginal smears), serological tests [ $\beta$-Dglucan ( $\beta$-DG), mannan, and anti-mannan antibodies], imaging tests (endoscopy, CT and MRI), and ophthalmological examination are useful for detecting systemic Candida disease $(2,3)$.

There are more than 150 species of Candida, 15 of which are isolated from patients as infectious agents with variable antifungal susceptibility (1). Candida albicans still remains the leading cause of Candida infections $(1,6)$. However, the

\footnotetext{
${ }^{1}$ Department of Hematology, Japan Community Healthcare Organization (JCHO) Tokyo Yamate Medical Center, Japan, ${ }^{2}$ Department of Gastroenterology, Japan Community Healthcare Organization (JCHO) Tokyo Yamate Medical Center, Japan, ${ }^{3}$ Department of Ophthalmology, Japan Community Healthcare Organization (JCHO) Tokyo Yamate Medical Center, Japan and ${ }^{4}$ Department of Pathology, Japan Community Healthcare Organization (JCHO) Tokyo Yamate Medical Center, Japan

Received for publication November 30, 2014; Accepted for publication April 1, 2015

Correspondence to Dr. Yukiko Komeno, ykomeno-tky@umin.ac.jp
} 

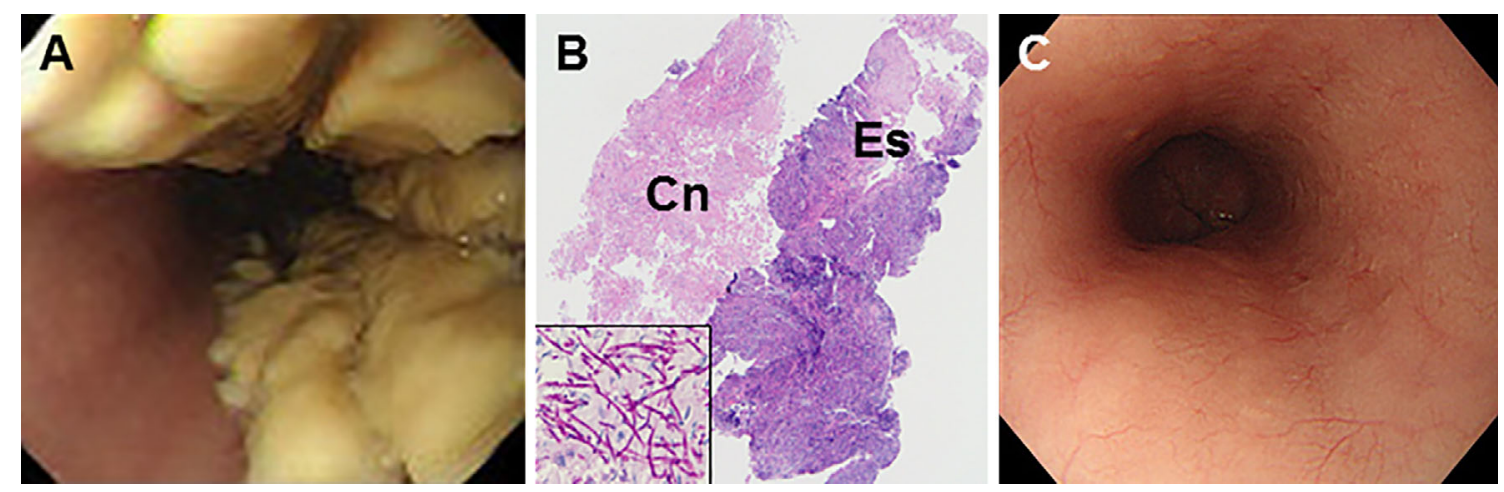

Figure 1. Esophageal candidiasis at the onset of AML. (A) A gastroendoscopy image of esophageal candidiasis in the mid-lower esophagus. (B) The histology of the biopsied esophageal candidiasis. A Hematoxylin and Eosin $(\mathrm{H} \& \mathrm{E})$ staining (magnification: $\times 20)$. Cn: a mass of candidiasis, Es: esophagus. Inset, a PAS stain of the mass (magnification: $\times 1,000) .(C)$ A gastroendoscopy image captured after the administration of induction chemotherapy and antifungal therapy.

frequency of infections by non-albicans Candida (NAC) species such as C. glabrata, C. krusei and C. parapsilosis, is increasing $(1,6)$. The isolation rates of those species depend on the features of the patients (age, disease, comorbidities) and local epidemics (1). In a recent prospective study in patients with hematological malignancies, candidemia was found to be predominantly caused by NAC species $(35 / 40$, $87.5 \%)$, particularly C. parapsilosis $(20 / 40,50 \%)(7)$.

Acute myeloid leukemia (AML) is a disease caused by the clonal expansion of myeloblasts in the peripheral blood, bone marrow or other tissues (8). It is a heterogeneous disease characterized by various chromosomal abnormalities and gene mutations (8). The typical clinical manifestations of AML are fever (neutropenia), fatigue (anemia) and bleeding (thrombocytopenia) caused by the expansion of blasts and decreased normal hematopoiesis in the bone marrow. Combination chemotherapy and hematopoietic stem cell transplantation are used in the treatment of AML. These therapies result in persistent neutropenia, which further increases the risk of opportunistic infection $(9,10)$.

We herein present the case of an AML patient whose initial manifestation was of persistent dysphagia (which continued for 5 weeks) caused by massive esophageal candidiasis. When patients with esophageal candidiasis have no past history of immunosuppressive therapies (chemotherapy, stem cell transplantation or steroid/immunosuppressant use) or HIV infection, hematological malignancy should be a differential diagnosis. This report also emphasizes the importance of thorough screening for Candida foci and the administration of systemic anti-fungal agents to prevent systemic invasive candidiasis in neutropenic patients with local candidiasis prior to or during chemotherapy.

\section{Case Report}

An otherwise healthy 47-year-old woman presented to a clinic with persistent dysphagia, cough and sputum. Antiulcer (lafutidine) and gastroprokinetic (mosapride) agents were prescribed, but did not alleviate her symptoms. She visited our hospital 2 weeks after the onset of symptoms. She did not have fever, shortness of breath, or an obvious bleeding tendency. Gastroendoscopy revealed the presence of multiple caseous lumps aligned vertically in the mid and lower esophagus (Fig. 1A). The mucosa of the oral cavity and upper esophagus was intact. Infection tests including tests for syphilis, hepatitis B virus, hepatitis C virus, and human immunodeficiency virus (HIV) were all negative. She refused to undergo additional blood tests on the same day. The clinical diagnosis of esophageal candidiasis was made, and oral miconazole gel $(2 \%, 20 \mathrm{~g} / \mathrm{day})$ was prescribed. The histology of the biopsied tissue showed a thick layer of epithelial cells containing numerous Candida hyphae and spores (Fig. 1B), and marked infiltration of inflammatory cells into the esophageal mucosa (data not shown). The findings were compatible with a diagnosis of esophageal candidiasis. The symptoms persisted for three additional weeks. She returned to our hospital with a high fever $\left(38^{\circ} \mathrm{C}\right)$. The patient's blood test results showed leukocytosis $(137,150 / \mu \mathrm{L}$; blasts, $85 \%)$, anemia and thrombocytopenia (Table). The patient was referred to the Department of Hematology and admitted to hospital. Multiple petechiae were observed on her chest and lower extremities. Her chest and abdominal $\mathrm{X}$ rays were normal. The diagnosis of AML without maturation was made by bone marrow aspiration and biopsy (Fig. 2) (8). The patient's blasts were positive for CD13, CD33, CD34, human leukocyte antigen (HLA)-DR and CD7, and negative for CD2, CD3, CD10, CD19 and CD20, and a del (9) chromosomal abnormality was detected (46, XX, del(9)(p?), 20 out of 20 cells). Intravenous doripenem ( $3 \mathrm{~g} /$ day, for febrile neutropenia), oral polymyxin B (PL-G, 3 million units/day, for gastrointestinal disinfection) and oral itraconazole (1\% oral solution, $20 \mathrm{ml} / \mathrm{day}$, for esophageal and potential systemic candidiasis) were administered (Day -2). The patient was catheterized with a peripherally inserted central catheter (PICC), and intravenous micafungin (150 mg/day) was added due to a higher fever (Day -1). The next day, induc- 
Table. Laboratory Test Results on Admission.

\begin{tabular}{|c|c|c|c|c|c|c|c|}
\hline & & Unit & Reference range & & & Unit & Reference range \\
\hline WBC & 137,150 & $/ \mu \mathrm{L}$ & $3,500-9,000$ & $\underline{\mathrm{ALT}}$ & $\underline{139}$ & $\mathrm{IU} / \mathrm{L}$ & $4-30$ \\
\hline Hemoglobin & $\underline{8.6}$ & $\mathrm{~g} / \mathrm{dL}$ & $12.0-16.0$ & $\underline{\mathrm{LDH}}$ & $\underline{862}$ & $\mathrm{IU} / \mathrm{L}$ & $100-230$ \\
\hline $\mathrm{MCV}$ & 96.6 & $\mathrm{fL}$ & $80.0-100.0$ & $\underline{\text { ALP }}$ & $\underline{619}$ & $\mathrm{IU} / \mathrm{L}$ & $100-328$ \\
\hline Platelet & $\underline{1.5}$ & $\times 10^{4} / \mu \mathrm{L}$ & $12.0-36.0$ & $\overline{\gamma-G T P}$ & 211 & $\mathrm{IU} / \mathrm{L}$ & $7-34$ \\
\hline \multicolumn{4}{|c|}{ Differential count of WBC } & Total-Bil & 0.9 & $\mathrm{mg} / \mathrm{dL}$ & $0.2-1.2$ \\
\hline Monocyte & 10.0 & $\%$ & $2.0-11.0$ & Amylase & $\underline{159}$ & $\mathrm{IU} / \mathrm{L}$ & $30-120$ \\
\hline Lymphocyte & $\underline{3.5}$ & $\%$ & $19.0-49.0$ & $\underline{\mathrm{BUN}}$ & $\underline{5}$ & $\mathrm{mg} / \mathrm{dL}$ & $8-20$ \\
\hline Basophil & 0.0 & $\%$ & $0.0-3.0$ & Creatinine & 0.7 & $\mathrm{mg} / \mathrm{dL}$ & $0.5-0.9$ \\
\hline Eosinophil & 1.5 & $\%$ & $0.0-5.0$ & CRP & $\underline{8.0}$ & $\mathrm{mg} / \mathrm{dL}$ & $0.0-0.4$ \\
\hline Neutrophil & $\underline{0.0}$ & $\%$ & $37.0-72.0$ & $\beta$-D-glucan & $\leq 4$ & $\mathrm{pg} / \mathrm{mL}$ & $<11.0$ \\
\hline \multirow[t]{2}{*}{$\underline{\text { Blast }}$} & $\underline{85.0}$ & $\%$ & - & Candida mannan Ag & $(-)$ & & \\
\hline & & & & Aspergillus Ag & $(-)$ & & \\
\hline Total protein & 7.8 & $\mathrm{~g} / \mathrm{dL}$ & $6.5-8.0$ & Procalcitonin & $<0.5$ & $\mathrm{ng} / \mathrm{mL}$ & $<0.5$ \\
\hline $\mathrm{AST}$ & 121 & IU/L & $10-33$ & T-Spot. TB & $(-)$ & & $(-)$ \\
\hline
\end{tabular}

Underlines show the values outside the reference ranges. Ag: antigen, ALP: alkaline phosphatase, ALT: alanine aminotransferase, AST: aspartate aminotransferase, BUN: blood urea nitrogen, CRP: C-reactive protein, $\gamma$-GTP: gamma-glutamyl transferase, LDH: lactate dehydrogenase, MCV: mean corpuscular volume
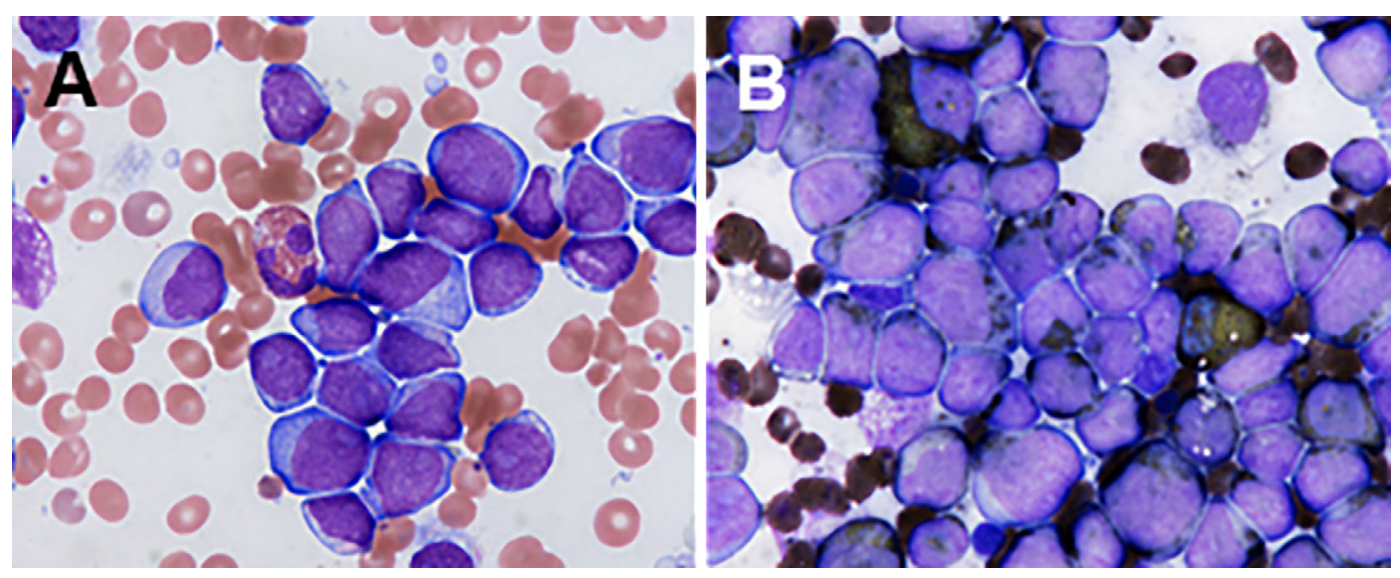

Figure 2. Bone marrow smears at diagnosis. (A) A H\&E staining (magnification: $\times 1,000)$. (B) A myeloperoxydase (MPO) staining (magnification: $\times 1,000)$. The blasts are positive for MPO.

tion chemotherapy was initiated with the administration of cytarabine $\left(100 \mathrm{mg} / \mathrm{m}^{2}\right)$ for 5 days and idarubicin $(12 \mathrm{mg} /$ $\mathrm{m}^{2}$ ) for 3 days (Day 1, Fig. 3) (10). The duration of cytarabine administration was shortened from 7 days to 5 days due to a rapid decrease of the patient's WBC count. $\beta$-DG, Candida mannan antigen, procalcitonin and one set of blood cultures performed before the initiation of antimicrobial therapy were all negative (Table). None of the blood cultures performed after the initiation of antimicrobial therapy turned positive (Fig. 3). Cultures of sputum, throat swab, feces and urine, which were sampled on day -1 and day 3, were found to all be negative (except for normal flora). On day 5, the PICC was removed and cultured; it was also found to be negative. From day 6 , granulocyte-colony stimulating factor (G-CSF, $300 \mu \mathrm{g} / \mathrm{day}$, drip infusion) was administered. The patient achieved complete remission of leukemia. The follow-up gastroendoscopy revealed no signs of candidiasis (Fig. 1C). During her recovery from the nadir, the patient complained of foggy vision in the right eye. An eye examination showed not only retinal hemorrhages in the right eye but also soft exudate in both eyes which were sug- gestive of ocular candidiasis (Fig. 4A). An eye examination after the administration of the first consolidation chemotherapy revealed no residual ocular candidiasis disease (Fig. 4B).

\section{Discussion}

We herein reported the case of a patient with AML whose initial clinical manifestation was of massive esophageal candidiasis. To the authors' knowledge, this is the first report to show afebrile and persistent esophageal candidiasis as the initial manifestation of AML in a patient without a history of immunosuppressive treatment. Although the subtype of Candida was not evaluated at the first endoscopic examination, it was sensitive to the intravenous administration of micafungin and oral itraconazole. In general, fungi are rarely identified as the cause of first fever early in the course of neutropenia (9). Rather, fungal infections are encountered after the first week of prolonged neutropenia (due to chemotherapy in the majority of cases) and empirical antibiotic therapy (9). Deep-tissue candidiasis (hepatic or hepa- 


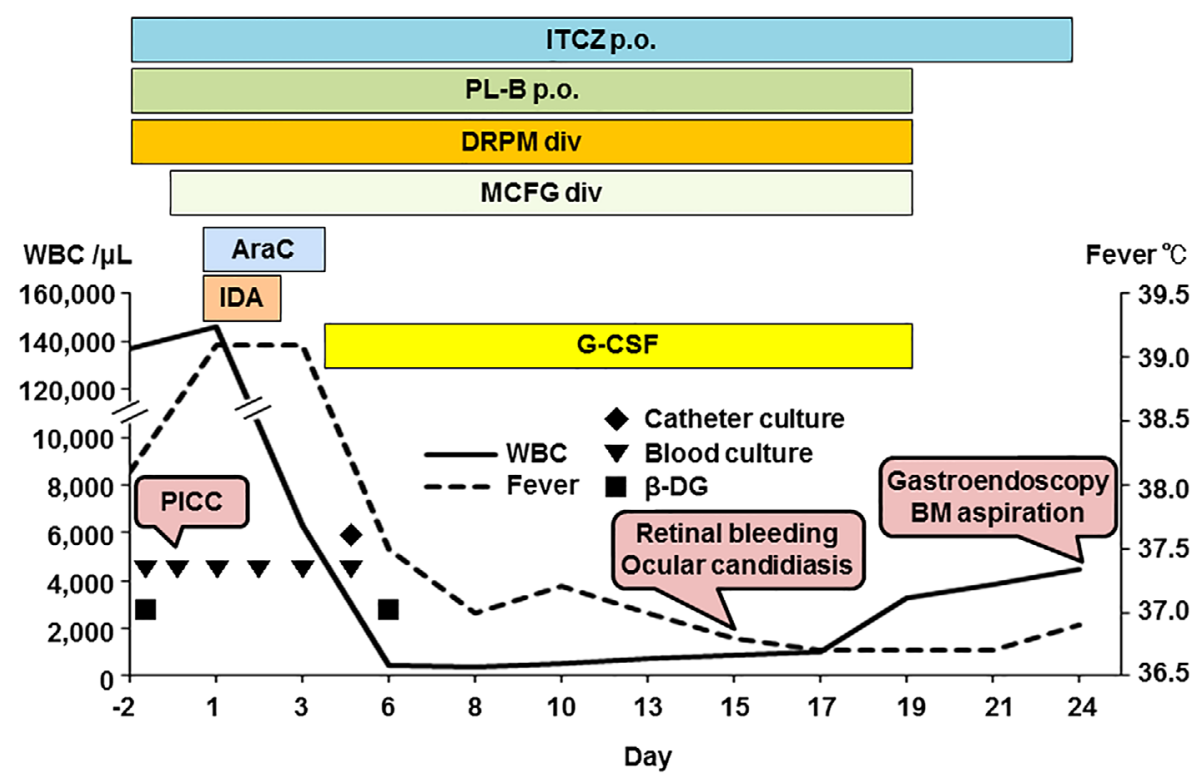

Figure 3. The clinical course of induction chemotherapy and antimicrobial therapy. The first day of chemotherapy was defined as day 1 . AraC: cytarabine, $\beta$-DG: $\beta$-D-glucan, BM: bone marrow, div: intravenous drip infusion, DRPM: doripenem, G-CSF: granulocyte-colony stimulating factor, IDA: idarubicin, ITCZ: itraconazole, MCFG: micafungin, PICC: peripherally inserted central catheter, PL-B: polymyxin B, p.o.: oral administration

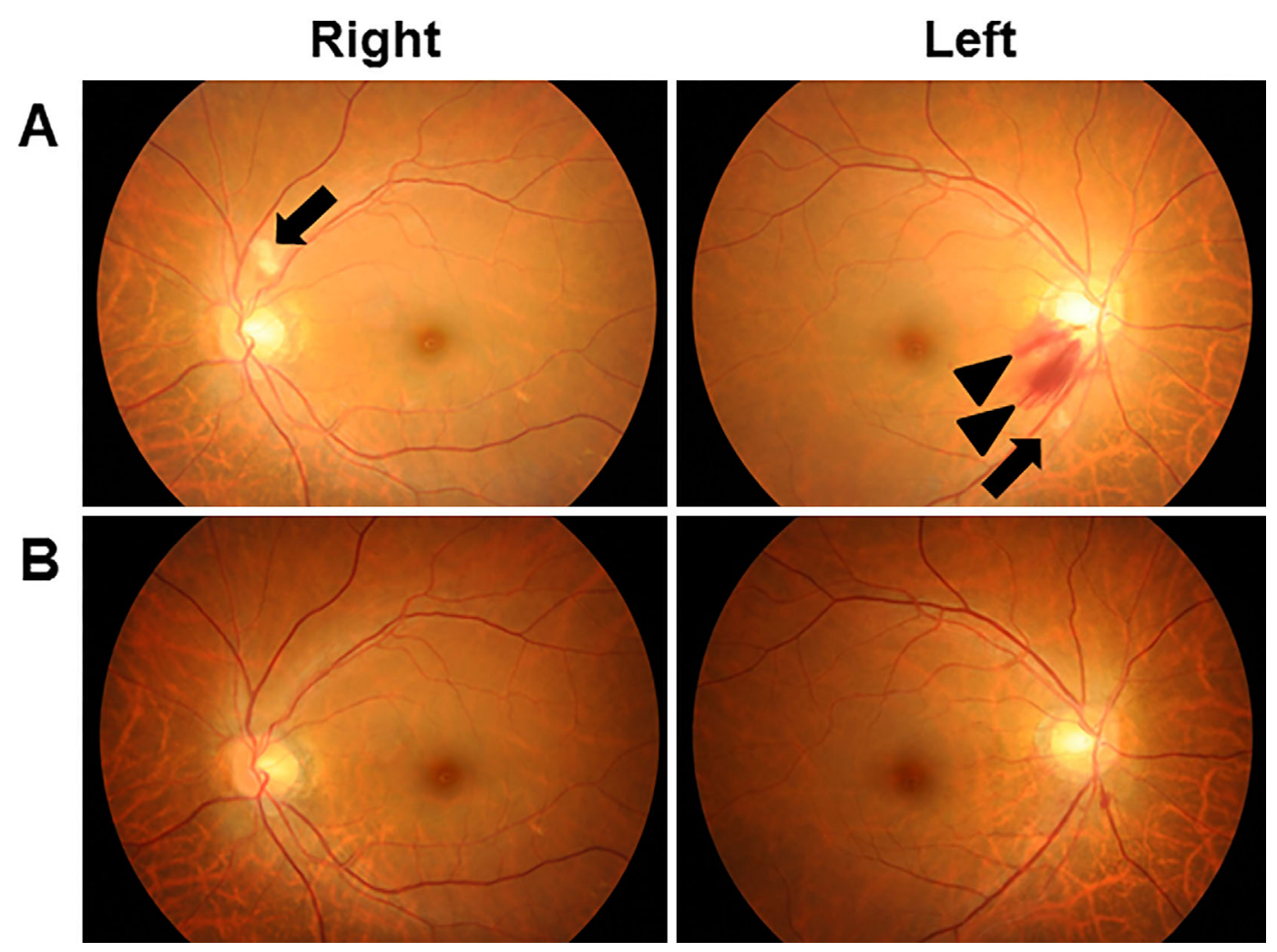

Figure 4. Bilateral ocular candidiasis. (A) Ocular fundi on day 15. Soft exudate suggestive of candidiasis (arrows) and retinal hemorrhages (arrowheads). (B) Ocular fundi after the administration of consolidation chemotherapy.

tosplenic disease, esophagitis, or endocarditis) is much less common (9). This is why the guidelines for the management of neutropenic fever recommend the use of antibiotics first, with the addition of antifungal agents if antibiotic therapy is not effective within 4-7 days (9). It should be noted that, at the present time, most patients with hematological malignancies or cancers undergoing chemotherapy take fluconazole for antifungal prophylaxis (9). In addition, an increasing 
number of clinical trials have reported to have optimized "prophylaxis" for invasive fungal infections (11). Thus, we demonstrated that afebrile Candida infection can be the sole initial manifestation of systemic disorders including hematological malignancies.

In this patient, esophageal candidiasis had been treated as peptic ulceration with esophagitis until gastroendoscopy was performed. The patient's condition was not accompanied by oral candidiasis, which is readily recognized and which can prompt physicians to perform gastroendoscopy to detect esophageal candidiasis. Interestingly, even among HIV patients with esophageal candidiasis, $56.5 \%$ of patients show no gastrointestinal symptoms, and $55.3 \%$ have no oral candidiasis (2). These data and our experience suggest that oral and esophageal candidiasis do not always coexist and that the decision to perform gastroendoscopy based on symptoms alone may lead to the missed or delayed diagnosis of esophageal candidiasis (2). Disease distribution (oral, esophageal or both) may depend on the local disruption of the mucosal barrier (e.g., reflux esophagitis and gingivitis) and the presence of Candida colonization. Candida colonization in the digestive system is associated with a higher incidence of candidemia (12). Multiple experimental models have demonstrated that not only Candida colonization but also mucosal damage are required to cause candidemia $(13,14)$.

The patient had an asymptomatic soft exudate suggestive of ocular candidiasis (Candida endophthalmitis) in both eyes, which might have become more severe without the intravenous administration of micafungin. The Infectious Diseases Society of America (IDSA) guidelines for the management of candidiasis recommend that all patients with candidemia undergo a dilated ophthalmological evaluation to exclude Candida endophthalmitis after the recovery of their neutrophil count (3). It is likely that candidemia caused ocular candidiasis in our patient, despite the negative result on all of the blood cultures and serological tests (including $\beta$ DG and Candida mannan antigen). This is compatible with the fact that the majority of patients who develop fever during neutropenia have no identifiable site of infection and no positive culture results (9).

The patient refused to undergo additional blood tests when the diagnosis of esophageal candidiasis was made, which delayed the diagnosis of AML. Earlier diagnosis of AML might have led to the earlier provision of systemic antifungal treatment and prevention of systemic candidiasis. It should be kept in mind that esophageal candidiasis is an AIDS-defining condition (2), and that when the condition is encountered, there should be an underlying immunodeficiency, especially cellular immunity.

How and when did this "neutropenic" AML patient develop esophageal candidiasis, candidemia and ocular candidiasis? It is reported that patients with congenital or acquired defects in cell-mediated immunity are particularly prone to mucocutaneous candidiasis, whereas systemic candidiasis is more commonly associated with neutropenia or congenital defects that affect neutrophil function (15). The patient did not have an HIV infection and did not have a history of cytotoxic/immunosuppressive therapy. Although her CD4+ cell count was not determined before chemotherapy, there were no other symptoms or test results suggestive of defective acquired cellular immunity, such as cytomegalovirus infection, tuberculosis, or herpes zoster (16). Based on these facts, AML is the only underlying disease to have triggered candidiasis in this patient. We are unsure as to when candidemia and ocular candidiasis developed because all the cultures and serum tests for Candida were negative (Fig. 3). The conditions likely developed before hospitalization, possibly when the patient became febrile, because systemic antifungals were administered on admission. Her fever might have been due to bacteremia, fungemia or both. The patient's ocular candidiasis was asymptomatic, because the lesions were off the optic papilla (Fig. 4). It is reasonable to think that the entry site of Candida into the bloodstream was the esophagus, because a biopsy of the esophagus demonstrated erosion of the epithelium under the Candida mass (Fig. 1B). Pre-existing erosion due to reflux esophagitis might have facilitated the Candida colonization to the lower esophagus, with the formation of huge lumps spreading upward. The patient was catheterized with a PICC after the administration of systemic antifungals. The cultures of catheter (removed on day 5 of chemotherapy) and diarrhea (which manifested at admission) were negative. These facts suggest that the PICC insertion site and the intestine/colon are less likely to be the Candida entry sites.

In summary, we reported the case of a patient with afebrile esophageal candidiasis as the initial manifestation of AML. Esophageal candidiasis suggests the presence of impaired immunity including AML. In neutropenic patients showing gastrointestinal symptoms, gastroendoscopy is recommended to find esophageal candidiasis, even in the absence of oral lesions. When patients have local candidiasis, the prompt screening of infection foci (blood tests, ophthalmological examination, CT and endoscopy) and systemic antifungal treatment should be considered to prevent progression to systemic invasive candidiasis, especially in neutropenic patients.

The authors state that they have no Conflict of Interest (COI).

\section{Acknowledgement}

We thank the patient for her valuable cooperation.

\section{References}

1. Yapar N. Epidemiology and risk factors for invasive candidiasis. Ther Clin Risk Manag 10: 95-105, 2014.

2. Nishimura S, Nagata N, Shimbo T, et al. Factors associated with esophageal candidiasis and its endoscopic severity in the era of antiretroviral therapy. PLoS One 8: e58217, 2013.

3. Pappas PG, Kauffman CA, Andes D, et al. Clinical practice guidelines for the management of candidiasis: 2009 update by the Infectious Diseases Society of America. Clin Infect Dis 48: 503-535, 2009. 
4. Lionakis MS. New insights into innate immune control of systemic candidiasis. Med Mycol 52: 555-564, 2014.

5. Dineshshankar J, Sivakumar M, Karthikeyan M, Udayakumar P, Shanmugam KT, Kesavan G. Immunology of oral candidiasis. J Pharm Bioallied Sci 6: S9-S12, 2014.

6. Papon N, Courdavault V, Clastre M, Bennett RJ. Emerging and emerged pathogenic Candida species: beyond the Candida albicans paradigm. PLoS Pathog 9: e1003550, 2013.

7. Gamaletsou MN, Walsh TJ, Zaoutis T, et al. A prospective, cohort, multicentre study of candidaemia in hospitalized adult patients with haematological malignancies. Clin Microbiol Infect 20: O50O57, 2014.

8. WHO Classification of Tumours of Haematopoietic and Lymphoid Tissues. 4th ed. Swerdlow SH, Campo E, Harris NL, et al, Eds. IARC, Lyon, 2008: 130-139.

9. Freifeld AG, Bow EJ, Sepkowitz KA, et al. Clinical practice guideline for the use of antimicrobial agents in neutropenic patients with cancer: 2010 Update by the Infectious Diseases Society of America. Clin Infect Dis 52: 427-431, 2011.

10. Ohtake S, Miyawaki S, Fujita H, et al. Randomized study of in- duction therapy comparing standard-dose idarubicin with highdose daunorubicin in adult patients with previously untreated acute myeloid leukemia: the JALSG AML201 Study. Blood 117: 2358-2365, 2011.

11. Nachbaur D, Angelova O, Orth-Holler D, et al. Primary antifungal prophylaxis with micafungin in patients with haematological malignancies: real-life data from a retrospective single-centre observational study. Eur J Haematol 94: 258-264, 2014.

12. Nucci M, Anaissie E. Revisiting the source of candidemia: skin or gut? Clin Infect Dis 33: 1959-1967, 2001.

13. Maccallum DM. Hosting infection: experimental models to assay Candida virulence. Int J Microbiol 2012: 363764, 2012.

14. Yan L, Yang C, Tang J. Disruption of the intestinal mucosal barrier in Candida albicans infections. Microbiol Res 168: 389-395, 2013.

15. Ashman RB, Farah CS, Wanasaengsakul S, Hu Y, Pang G, Clancy RL. Innate versus adaptive immunity in Candida albicans infection. Immunol Cell Biol 82: 196-204, 2004.

16. Lucas S, Nelson AM. HIV and the spectrum of human disease. J Pathol 235: 229-241, 2015.

(C) 2015 The Japanese Society of Internal Medicine http://www.naika.or.jp/imonline/index.html 